\title{
Exploração da noção de função em tabelas e gráficos por alunos do $3 .^{\circ}$ ciclo Exploration of the concept of function in tables and graphs by students of the 3rd cycle
}

\author{
Floriano Viseu, José António Fernandes, Paula Mendes Martins \\ Universidade do Minho
}

\begin{abstract}
Resumo
Atendendo ao papel que as diferentes representações de uma função desempenham na sua aprendizagem, procuramos averiguar a noção de função de alunos do $3 .^{\circ}$ ciclo através de situações representadas por gráficos e por tabelas. Adotamos uma abordagem qualitativa na procura de compreender o que os alunos expressam nas suas justificações. Os alunos do $7 .^{\circ}$ ano apresentam uma noção mais clara de função do que os restantes alunos, e em ambas as representações estudadas. Adicionalmente, observa-se uma tendência de maior acerto na identificação de situações que não são funções do que no caso de situações que são funções.
\end{abstract}

Palavras-chave: aprendizagem, função, tabelas, gráficos, alunos do $3 .^{\circ}$ ciclo

\begin{abstract}
Given the role that the different representations of a function play in their learning, we seek to ascertain the notion of the function by students of the 3rd cycle considering situations represented by graphs and tables. A qualitative approach was adopted in the search to understand the students' justifications. The students of the 7 th year present a clearer notion of function than the other students, and in both representations studied. In addition, there is a trend towards greater accuracy in identifying situations that are not functions than in situations that are functions.

Keywords: learning, function, tables, graphs, 3rd cycle students
\end{abstract}

$\mathrm{Na}$ aprendizagem de conteúdos matemáticos, geralmente trabalha-se do concreto para o abstrato, procurando-se acompanhar o desenvolvimento cognitivo do aluno. Paulatinamente, até ao final do $2 .^{\circ}$ ciclo, o raciocínio abstrato surge sobretudo na generalização das propriedades das operações numéricas e no estudo de sequências e regularidades. É na transição para o $3 .^{\circ}$ ciclo que se incrementa o desenvolvimento do raciocínio abstrato. Para esse desenvolvimento muito contribui o estudo do tópico de Funções, que surge pela primeira vez no programa do $7 .^{\circ}$ ano de escolaridade. A partir deste ano de escolaridade, as funções têm expressão significativa no currículo quer do $3 .^{\circ}$ ciclo quer do secundário. Em ambos os ciclos de escolaridade, as recomendações metodológicas atuais para o estudo de funções destacam o recurso a diferentes representações, tais como a tabelar, a gráfica e a simbólica. O recurso a diferentes representações no estudo das funções promove, segundo Chazan e Yerushalmy (2003), uma melhor compreensão por parte dos alunos deste tópico. Para que isso aconteça, importa que as estratégias de ensino valorizem a conexão ente as diferentes representações e que atendam ao grau de maturidade cognitiva do aluno. Partindo destes pressupostos, procuramos averiguar a noção de função de alunos do $3 .^{\circ}$ ciclo através de situações representadas por gráficos e por tabelas.

\section{As funções no currículo do $3 .^{\circ}$ ciclo}

O tópico de funções surge pela primeira vez no programa do $7 .^{\circ}$ ano no domínio 'Funções, Sequências e Sucessões' (MEC, 2013). Neste ano de escolaridade, começa-se por distinguir correspondências entre conjuntos que traduzem funções, adquirir a terminologia própria de funções, no que respeita à identificação do objeto, imagem, domínio, conjunto de chegada, contradomínio e variáveis independente e dependente. Segue-se a definição de igualdade de funções, a representação de funções por meio de diagramas de setas, tabelas e gráficos cartesianos e as operações com funções, com o intuito de introduzir as noções de função linear e de função fim e, posteriormente, a noção de função de proporcionalidade direta.

No $8 .^{\circ}$ ano de escolaridade estudam-se os gráficos de funções afins, reconhecendo o gráfico de uma função afim através da translação do gráfico de uma função linear com o mesmo declive, o significado dos parâmetros da equação da reta que representa uma função afim e o procedimento para se obter o declive dessa reta conhecendo dois pontos quaisquer do domínio da função.

No 9. ${ }^{\circ}$ ano de escolaridade estudam-se as funções de proporcionalidade inversa, através das representações tabelar, analítica e gráfica, e reconhece-se a curva que representa funções do tipo $f(x)=a x^{2}(\operatorname{com} a \neq 0)$ e o conjunto-solução da equação do 2. ${ }^{\circ}$ grau $a x^{2}+b x+c=0($ com $a \neq 0)$ como sendo a intersecção da parábola de equação $y=a x^{2}$ com a reta de equação $y=-b x-c$. 
Com os conhecimentos adquiridos em cada um dos anos de escolaridade do $3 .^{\circ}$ ciclo, pretende-se que os alunos os apliquem na resolução de problemas em contextos variados.

\section{As representações no ensino de funções}

As diferentes representações dos conceitos matemáticos revestem-se de grande importância na aprendizagem dos alunos (NCTM, 2007) pois cada uma dessas representações acrescenta ou destaca algo que é oculto ou pouco saliente nas outras representações. Assim, a exploração das diferentes representações na aprendizagem assume-se como um requisito para uma aprendizagem mais profunda.

No caso do conceito de função, destacam-se as representações tabelares, gráficas, algébricas e diagramáticas, cada uma das quais revela aspetos e propriedades específicas das funções, incluindo também as suas diferentes aplicações.

Representação tabelar. Esta representação, também dita representação numérica, salienta vários pares de valores das variáveis envolvidas na relação funcional e facilita a generalização no sentido de descobrir uma lei de formação desses valores, que é característica da representação algébrica. Nesta representação, a verificação de que se trata de uma função requer que o aluno analise os valores numéricos que são fornecidos na tabela, segundo a relação em questão. Para Brown e Mehilos (2010), a representação tabelar facilita a passagem do concreto para o abstrato, dando significado às variáveis e às expressões algébricas.

Representação gráfica. Esta representação consiste em representar todos os pontos cujas coordenadas verificam a relação funcional em questão. Comparativamente com outras representações, esta revela certas propriedades das funções, como sejam os zeros, o sinal, a monotonia, etc. Já em termos de averiguar se uma relação é uma função, adota-se como critério o facto de qualquer reta horizontal intercetar o gráfico no máximo num ponto. Segundo Friedlander e Tabach (2001), a representação gráfica é intuitiva e apelativa, devido ao seu carácter visual.

Representação algébrica. Esta representação, para além dos conjuntos implicados na função, envolve uma relação algébrica entre as variáveis consideradas na função. Trata-se de uma representação altamente compactada e abstrata, em que a lei algébrica desempenha um papel fundamental no estudo da função, requerendo que o aluno manipule algebricamente a relação para estudar propriedades da função, incluindo a verificação de que se trata de uma função. Para Friedlander e Tabach (2001), a representação algébrica é precisa, geral e efetiva na apresentação de padrões e modelos matemáticos, sendo muitas vezes a única forma de justificar afirmações gerais.

Representação diagramática. Na representação de funções através de diagramas sagitais destacam-se os diferentes elementos da definição de uma função: o domínio, o conjunto de chegada e o contradomínio e a correspondência entre objetos e imagens. Simultaneamente, trata-se de uma representação abstrata e mais genérica do que as anteriores, podendo aplicar-se a qualquer tipo de entes e não apenas a números, e sendo frequentemente usada para introduzir aos alunos, pela primeira vez, a noção de função. Assim, a introdução da noção de função a partir desta representação corresponde a uma valorização da abordagem formal do conceito em detrimento do seu significado.

Santos e Barbosa (2016, 2017) advogam que a complementaridade dos aspetos revelados pelas diferentes representações do conceito de função requer que os alunos explorem essas diferentes representações na sua aprendizagem desde o $7 .^{\circ}$ ao $12 .^{\circ}$ ano (MEC, 2013, 2014). Nachlieli e Tabach (2012) referem que nem sempre isso acontece, visto que, frequentemente, é abordada apenas uma das representações ou são abordadas mais do que uma representação, mas sem as relacionar. Para Triphathy (2008), limitar o estudo da noção de função a uma representação é "abordar o conceito de olhos vendados" (p. 438).

O presente estudo foca-se na definição de função, sendo pedido aos alunos que classifiquem em função ou não função relações representadas sob a forma tabelar e gráfica.

\section{Método}

Com o objetivo de averiguar a noção de função de alunos do $3 .^{\circ}$ ciclo, apresentamos várias situações representando relações entre duas variáveis através de gráficos e tabelas, tendo em vista comparar o desempenho dos alunos ao longo dos diferentes anos deste ciclo. Em termos curriculares, os alunos do $3 .^{\circ}$ ciclo estudam conteúdos de funções através das suas diferentes representações em cada um dos anos deste ciclo escolar. Mais concretamente, no $7 .^{\circ}$ ano é introduzida pela primeira vez a noção de função, prosseguindo o estudo da função afim no $8 .^{\circ}$ ano e da função de proporcionalidade inversa e da função quadrática do tipo $f(x)=a x^{2}\left(\operatorname{com}(\operatorname{com} a \neq 0)\right.$ no $9 .^{\circ}$ ano. Para tal, recolhemos dados de alunos do $7 .^{\circ}$ ano $(n=30)$, do $8 .^{\circ}$ ano $(n=23)$ e do $9 .^{\circ}$ ano $(n=27)$, que integravam turmas de diferentes escolas de uma cidade do norte de Portugal.

Propôs-se aos alunos uma tarefa composta por cinco itens, em que lhes era pedido que identificassem se cada situação representava, ou não, uma função (Figura 1). 
Indica quais das seguintes situações representam, ou não, uma função. Justifica a tua resposta.

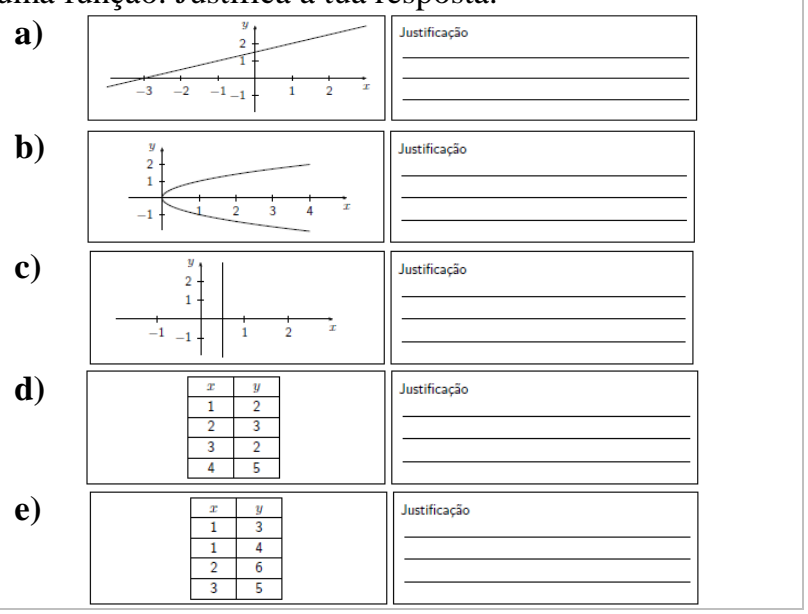

Figura 1: Tarefa proposta aos alunos.

Pelo enunciado da tarefa, verificamos que os itens a), b) e c) envolvem a representação de relações através de gráficos e os itens d) e e) envolvem a representação de relações através de tabelas. Em qualquer um destes itens, o aluno deveria concluir se a situação correspondia a uma função, ou não, apresentando também uma justificação para a sua resposta.

As respostas dos alunos foram classificadas em corretas (C), parcialmente corretas (PC) e incorretas (I). Em cada item, só se consideraram $\mathrm{C}$ as respostas que, além de classificarem corretamente a relação em função ou não função, apresentavam também uma justificação adequada. Nos casos em que consideravam corretamente que o item apresentado é, ou não, função mas sem justificação ou com justificação incorreta ou insuficiente considerou-se a resposta como PC. Além disso, consideraram-se as frequências dos diferentes tipos de respostas nos diferentes itens e segundo o ano de escolaridade e o tipo de representação.

\section{Análises de dados}

Na Tabela 1 encontram-se registadas as respostas dos alunos aos diferentes itens da tarefa proposta, segundo os diferentes tipos de resposta considerados (C, PC, I) e incluindo também as não respostas (NR). Globalmente, no conjunto dos cinco itens, obteve-se $25 \%$ de respostas C, $35 \%$ de respostas PC, $16,5 \%$ de respostas I e $23,5 \%$ NR. Destes resultados, salienta-se uma considerável dificuldade dos alunos em identificar funções.

\section{Tabela 1}

Frequência dos diferentes tipos de resposta dos alunos a cada um dos itens da tarefa $(n=80)$.

\begin{tabular}{|c|c|c|c|c|c|c|c|c|c|c|c|c|c|c|c|}
\hline \multirow{3}{*}{$\begin{array}{l}\text { Tipo de } \\
\text { resposta }\end{array}$} & \multicolumn{9}{|c|}{ Representaçăo Gráfica } & \multicolumn{6}{|c|}{ Represertaçăo Tabelar } \\
\hline & \multicolumn{3}{|c|}{ a) } & \multicolumn{3}{|c|}{ b) } & \multicolumn{3}{|c|}{ c) } & \multicolumn{3}{|c|}{ d) } & \multicolumn{3}{|c|}{ e) } \\
\hline & $7^{\circ}$ & $8^{\circ}$ & $9^{\circ}$ & $7^{\circ}$ & $8^{\circ}$ & $9^{\circ}$ & $7^{\circ}$ & $8^{\circ}$ & $9^{\circ}$ & $7^{\circ}$ & $8^{\circ}$ & $9^{\circ}$ & $7^{\circ}$ & $8^{\circ}$ & $9^{\circ}$ \\
\hline $\mathrm{C}$ & 10 & 2 & 2 & 10 & 1 & 3 & 12 & 5 & 4 & 14 & 1 & 3 & 19 & 5 & 9 \\
\hline PC & 13 & 16 & 17 & 12 & 7 & 9 & 9 & 4 & 11 & 9 & 11 & 4 & 4 & 4 & 10 \\
\hline I & 2 & 1 & - & 3 & 5 & 7 & 6 & 4 & 7 & 5 & 5 & 11 & 4 & 5 & 1 \\
\hline NR & 5 & 4 & 8 & 5 & 10 & 8 & 3 & 10 & 5 & 2 & 6 & 9 & 3 & 9 & 7 \\
\hline
\end{tabular}

Seguidamente, na Tabela 2, aprofunda-se a análise através das variáveis ano escolar e tipo de representação. Em termos dos três anos escolares envolvidos no estudo, constata-se que, na globalidade dos cinco itens, são os alunos do $7 .^{\circ}$ ano que apresentam um maior número de respostas corretas, enquanto os alunos do $8 .^{\circ}$ e do $9 .^{\circ}$ anos se destacam nas respostas PC, I e NR. Estes resultados indiciam que são os alunos do $7 .^{\circ}$ ano que têm uma noção mais precisa de função.

Tabela 2

Percentag em dos diferentes tipos de resposta em cada um dos anos de escolaridade e em cada tipo de representação $(\mathrm{n}=80)$.

\begin{tabular}{lccccccc}
\hline & \multicolumn{3}{c}{ Ano escolar } & & \multicolumn{2}{c}{ Tipo de representação } \\
\cline { 2 - 4 } \cline { 7 - 8 } Tipo de resposta & $\mathbf{7}^{\mathbf{0}}$ & $\mathbf{8}^{\circ}$ & $\mathbf{9}^{\circ}$ & & Gráfica & Tabdar \\
\hline C & 43,3 & 12,2 & 15,5 & & 20,4 & 31,9 \\
PC & 31,3 & 36,5 & 37,8 & & 40,8 & 26,2 \\
I & 13,4 & 17,4 & 19,3 & & 14,6 & 19,4 \\
NR & 12,0 & 33,9 & 27,4 & & 24,2 & 22,5 \\
\hline Nota: C-Corr eta; PC-Parcialm ente correta; I-Incorreta; NR-Năo resposta. & & &
\end{tabular}

Considerando o tipo de representação, verifica-se que na representação tabelar se obteve um maior número de respostas corretas do que na representação gráfica. Para esta discrepância contribuiu a não apresentação de qualquer justificação por alguns alunos no caso da representação gráfica, levando a classificar as suas respostas como PC.

$\mathrm{Na}$ continuação apresentam-se exemplos de respostas dos alunos nos diferentes itens, segundo a representação gráfica e a representação tabelar.

\section{Identificação de funções em representações gráficas}

Dos itens que traduzem relações através da representação gráfica, o item a) representa uma função porque, como exemplificam algumas das respostas dos alunos consideradas corretas: "cada objeto tem uma só imagem" (A5, $7 .^{\circ}$ ano); "cada $x$ corresponde um $y$ " (A47, 8. ano); "cada valor de $x$ tem um único resultado" (A80, 9. ${ }^{\circ}$ ano). O mesmo já não acontece com os itens b) e c). O item b) traduz uma relação que não é função porque "o objeto 1 tem duas imagens" (A8, 7. ${ }^{\circ}$ ano); "para cada $x$ corresponde mais do que um $y$ " (A47, 8. ${ }^{\circ}$ ano); "existe mais que um valor de $y$ para $x$ " (A62, 9. ${ }^{\circ}$ ano). Também o item c) traduz uma relação que não é função porque "o objeto 0.5 tem mais de uma imagem" (A17, $7 .^{\circ}$ ano); " $f(x)$ apresenta vários resultados para um só valor de $x$ " (A65, 9. ${ }^{\circ}$ ano).

Perante as justificações dadas, percebe-se que alguns alunos, de cada um dos níveis escolares em estudo, têm uma noção precisa de função. O mesmo já não dá para se perceber nas respostas consideradas parcialmente corretas. No caso do item a), alguns alunos respondem somente que a relação "É [uma função]" (A28, $7 .^{\circ}$ ano; A51, 8. ${ }^{\circ}$ ano; A67, 9..$^{\circ}$ ano), enquanto outros apresentam uma justificação inadequada dessa resposta, como são exemplo as seguintes afirmações: "É, porque a cada imagem corresponde um objeto" (A20, 7. ${ }^{\circ}$ ano; A49, 8. ano; A54, 9. ${ }^{\circ}$ ano); "É, porque o objeto tem a sua imagem" (A21, $7 .^{\circ}$ ano); "É, porque passa por $x$ e por $y$ " (A31, 8. ano); "Sim, porque apresenta uma constante" (A77, 9. ${ }^{\circ}$ ano)

O mesmo teor de respostas também se verificou nos itens b) e c). No item b), alguns alunos somente consideram que a situação representada "Não [é uma função]" (A19, $7 .^{\circ}$ ano; A71, 9. ${ }^{\circ}$ ano), enquanto outros consideram que não representa uma função "porque não é uma reta" (A2, 7. ${ }^{\circ}$ ano), ou "porque a reta nunca pode 
ser curva" (A40, 8. ano), ou "porque o eixo dos $x$ e dos $y$ estão trocados" (A67, 9. ${ }^{\circ}$ ano).

Relativamente ao item c), alguns alunos também só respondem que a situação traduzida "Não [é uma função]" (A19, 7. ${ }^{\circ}$ ano; A45, 8..$^{\circ}$ ano; A69, 9..$^{\circ}$ ano), enquanto outros consideram essa resposta porque "as retas que representam funções num gráfico são sempre perpendiculares à reta y ou paralela à reta $\mathrm{x}$ " $\left(\mathrm{A} 3,7 .^{\circ}\right.$ ano), ou "porque só passa pelo ponto $x \pm 0.5$ " (A43, 8. ano), ou "porque a função constante fica paralela ao eixo das ordenadas" (A71, 9. ${ }^{\circ}$ ano).

Quanto às respostas incorretas, no 7. ano há quem considere que a situação traduzida no item a) não é uma função "porque o gráfico é uma reta oblíqua" (A1), ou "porque a reta não passa no ponto de origem" (A29). Já no $8 .^{\circ}$ ano, somente um aluno considerou que a relação deste item não representa uma função "porque atravessa em linha reta dois números concretos" (A32).

No que diz respeito ao item b), algumas das respostas consideradas incorretas no $7 .^{\circ}$ ano deveram-se a considerar, inapropriadamente, que a relação representa uma função "porque cada um dos objetos pertence a uma imagem" (A25). Entre as respostas incorretas dos alunos do $8 .^{\circ}$ ano há quem refira que se trata de uma função "porque é uma função linear" (A36) ou "porque é uma parábola" (A52). Este tipo de justificação também foi dada por um aluno do 9..$^{\circ}$ ano, "é, porque é uma parábola" (A57), enquanto os restantes que responderam incorretamente também consideram tratar-se da representação de uma função "porque passa pela origem" (A72) ou "porque é uma função linear pois as suas retas passam nos eixos $x$ e $y$ " (A79).

Relativamente ao item c), as respostas incorretas apontam a situação como representando uma função porque, para os alunos do $7 .^{\circ}$ ano, "o gráfico apresenta uma reta vertical" (A2) ou "cada objeto tem a sua única imagem" (A7) ou por se tratar de "uma função constante, porque cada objeto tem sempre a mesma imagem" (A 27). Os alunos do $8 .^{\circ}$ e $9 .^{\circ}$ anos que responderam incorretamente também consideram que se trata de uma função, porque, para os alunos do $8 .^{\circ}$ ano, "é uma função constante" (A32) ou "é uma função afim" (A36), e, para os alunos do 9. ano, "é uma função do tipo constante" (A57) ou "é uma função constante pois ambas as retas vão na mesma direção logo são estritamente paralelas" (A79).

\section{Identificação de funções em representações tabelares}

Os restantes itens traduzem relações através da representação tabelar. No caso do item d), a relação é uma função, identificada como tal por alunos dos três anos, como exemplificam algumas das respostas dos alunos consideradas corretas: "a cada elemento $x$ corresponde um só de $y$ " (A24, $7 .^{\circ}$ ano); "a cada objeto corresponde apenas uma imagem" (A49, 8..$^{\circ}$ ano); "cada valor de $x$ tem um único valor $y$ " (A80, 9. ${ }^{\circ}$ ano). Já a situação apresentada no item e) não representa uma função, porque "o elemento 1 corresponde a dois elementos" (A27, 7. . ano); "um elemento de $x$ não pode ter mais que uma correspondência" (A33, 8. ${ }^{\circ}$ ano); "a mesma abcissa não pode ter diferentes ordenadas" (A73, 9. ${ }^{\circ}$ ano).
Quanto às respostas consideradas parcialmente corretas, no item d), enquanto alguns alunos somente referem que a relação "É [uma função]" (A30, 7. ${ }^{\circ}$ ano; A50, 8..$^{\circ}$ ano; A78, 9..$^{\circ}$ ano), outros justificam essa resposta dizendo que tal se deve a que "cada objeto tem a sua imagem" (A7, 7. ${ }^{\circ}$ ano), ou "todos têm correspondência" (A33, 8..$^{\circ}$ ano), ou "pode ser definida por uma expressão algébrica" (A61, 9. ${ }^{\circ}$ ano).

Nas respostas incorretas a este item há quem considere que a relação não traduz uma função porque "a diferença de 2 e 1 é diferente de 2 e 3 " (A2, $7^{\circ}$ ano), ou "não está representado num gráfico" (A32, $8 .^{\circ}$ ano), ou "a constante não é igual em todos os valores de x e y" (A66, 9. ${ }^{\circ}$ ano).

Relativamente ao item e), algumas das respostas parcialmente corretas somente referem que a relação "Não [é uma função]" (A19, 7. ano; A45, 8. ano; A69, 9. ${ }^{\circ}$ ano), enquanto outras apresentam justificações a essa resposta dizendo que tal se deve a que "a imagem se repete" (A30, 7..$^{\circ}$ ano), ou "porque não está representada por um gráfico" (A32, 8. ano) ou "porque a constante não é igual a todos" (A66, 9. ${ }^{\circ}$ ano).

Nas respostas consideradas incorretas a este item, alguns alunos referem tratar-se de uma função porque "o conjunto de partida tem um único objeto" (A15, 7. ano), ou porque "já temos as coordenadas" (A38, $8^{\circ}$ ano). O único aluno do $9 .^{\circ}$ ano que respondeu incorretamente refere apenas "Sim" (A78, 9. ${ }^{\circ}$ ano).

\section{Considerações finais}

Da análise das respostas dos alunos às relações apresentadas sob a forma de gráfico e tabela conclui-se que são os alunos do $7 .^{\circ}$ ano que revelam ter uma noção mais precisa do conceito de função. Tal facto indicia dever-se ao estudo recente deste tópico neste ano de escolaridade. A sistematicidade desse estudo nos seguintes anos de escolaridade tende a não fazer a diferença na identificação dos atributos essenciais do conceito de função, fortemente evidenciados no registo escrito ou na oralidade da expressão 'um e um só', nem em termos da exploração de diferentes representações, tais como diagramas, tabelas, gráficos e símbolos.

Em geral, no contexto escolar não são salientados os dois atributos essenciais do conceito de função: 1) trata-se de uma relação binária, que é 2) unívoca. Em geral, os professores e os manuais escolares referem que, numa função, a cada elemento $x$ corresponde um e a um só elemento ${ }^{y}$. Esta forma compactada da definição de função, em que não são explicitados os dois atributos da definição de função, constitui um obstáculo para os alunos.

No que respeita à explicitação dos atributos essenciais, os alunos do $7 .^{\circ}$ ano demarcam-se dos demais na relação existente entre os valores do domínio e do conjunto de chegada de uma dada correspondência, o que determina ou não a existência de uma função (Carraher \& Schliemann, 2007). Quanto às representações gráfica e tabelar, embora tendam a marcar presença nos manuais escolares e, supostamente, nas atividades da sala de aula, os alunos do $7 .^{\circ}$ ano revelam mais capacidade de aplicar a 
definição de uma função na análise da informação proveniente de cada uma dessas representações.

Em termos gerais, os alunos dos três anos de escolaridade sentem-se mais à-vontade em distinguir se uma dada relação é ou não função quando está em causa uma representação tabelar do que quando está em causa uma representação gráfica. Este resultado pode dever-se a uma maior utilização de tabelas nas estratégias de ensino do tópico Funções do que de gráficos, que exige outros requisitos na interpretação da informação para além da correspondência entre elementos, como, por exemplo, a relação entre pares ordenados e a distinção das variáveis. A dificuldade que os alunos revelam em conectar os atributos essenciais de uma função entre diferentes representações aponta para uma maior utilização de uma só representação quando estudam o tópico de Funções (Carraher \&Schliemann, 2007). Em termos gráficos, Markovits, Eylon e Bruckeimer (1998) consideram que os alunos tendem a manifestar uma conceção errada de linearidade, ficando com a ideia de que o gráfico de uma função é uma reta, o que pode dever-se à predominância no ensino de gráficos deste tipo. Neste estudo, tal conceção não se verifica, como comprova a similaridade entre o número de respostas corretas a uma relação traduzida por um gráfico de uma função afim e o número de respostas corretas à relação que traduz uma parábola representada na horizontal.

Para Duval (2006), a ligação das diferentes representações de funções não é fácil de efetuar. As representações só são mobilizadas e desenvolvidas se forem transformadas noutras, realçando desta forma a importância das conexões entre as várias representações para a melhor compreensão por parte dos alunos dos conceitos matemáticos. Através das várias representações, a compreensão do que é matematicamente importante numa representação ajuda a efetuar a sua conversão para outra forma de representação e a identificar a função a partir do conteúdo dessa mesma representação.

\section{Referências}

Brown, A. S. \& Mehilos, M. (2010). Using tables to bridge arithmetic and algebra. Mathematics Teaching in the Middle School, 15(9), 532-538.

Carraher, D. W. \& Schliemann, A. D. (2007). Early algebra and algebraic reasoning. In F. K. Lester (Ed.), Second Handbook of Research on Mathematics Teaching and Learning (Vol. 2, 669-705). Charlotte, N.C: Information Age Publishing, Inc. and NCTM.

Chazan, D. \& Yerushalmy, M. (2003). On appreciating the cognitive complexity of school algebra: research on algebra learning and directions of curricular change. In J. Kilpatrick, W. Martin, \& D. Schifter (Eds.), A research companion to principles and standards for school mathematics (pp. 123-135). Reston VA: NCTM.

Duval, R. (2006). A cognitive analysis of problems of comprehension in a learning of mathematics. Educational Studies in Mathematics, 61, 103-131.

Friedlander, A. \& Tabach, M. (2001). Promoting multiple representations in algebra. In A. A. Cuoco
(Ed.), The roles of representation in school mathematics (pp. 173-185). Reston, VA: NCTM.

Markovits, Z., Eylon, B. \& Bruckeimer, M. (1998) Difficulties students have with the function concept. In A. T. Coxford \& A. P. Shulte (Eds.), The ideas of algebra: K-12 (pp. 43-60). Reston, VA: National Council of Teachers of Mathematics.

Ministério da Educação e Ciência (2013). Programa de matemática para o ensino básico. Lisboa: Autor.

Ministério da Educação e Ciência (2014). Programa de Matemática A - Ensino secundário. Lisboa: Autor.

Nachlieli, T. \& Tabach, M. (2012). Growing mathematical objects in the classroom-the case of function. International Journal of Educational Research, 51/52, 10-27.

Santos, G. L. D. \& Barbosa, J. C. (2016). Um modelo teórico de matemática para o ensino do conceito de função a partir de um estudo com professores. UNIÓN, 48, 143-167.

Santos, G. L. D. \& Barbosa, J. C. (2017). Como ensinar o conceito de função. Educação Matemática em Revista, 22(53), 27-37.

Tripathi, P. N. (2008). Developing mathematical understanding through multiple representations. Mathematics Teaching in Middle School, 13(8), 438-353.

\section{Agradecimento}

Este trabalho é financiado pelo CIEd - Centro de Investigação em Educação, UID/CED/01661/, Instituto de Educação, Universidade do Minho, através de fundos nacionais da FCT/MCTES-PT 